This substance was mixed with milk and, after sterilizing, 5 c.c. of the suspension were injected into the peritoneal cavity of guinea-pigs and the animals killed from five hour's to twenty-one days later.

On account of the frequent presence of a spontaneous deposit of insoluble matter in the lungs of animals and the uncertainty which on that account might follow a microscopic examination of the tissues, a deposit sufficient for macroscopic recognition was deemed necessary for a definite conclusion.

I shall not in this preliminary notice give all the details of these experiments. The results do not differ materially from those obtained by Beitzke, except that the deposit, the color of which precluded confusion with a natural anthracosis, did not appear to extend beyond the lymph glands.

The blue matter was regularly taken up by the lymphaties of the mesentery and of the diaphragm. The network of glands surrounding the spleen and the liver was heavily loaded, although none could be observed on section of these organs. The diaphragm was within a few hours colored blue and the lymph ducts of the anterior and posterior mediastinum, as well as the glands lying beneath the sternum, were colored blue and could be traced to the root of the neck.

The bronchial glands were in some casos stained blue and in others not. The lungs often showed a peculiar motiled appearance, but this could not be identified as due to the ultramarine blue.

The staining of the bronchial glands may perhaps indicate that the substance has been reabsorbed from the lungs, especially as the larger lymph vessels of the thorax were shown to be conveying the substance to the veins returning to the right heart, but it may also indicate merely that on account of abnormalities in the lymph ducts a closer relationship between the anterior and posterior ducts and the bronchial glands existed in these particular animals.

It was remarkable that, considering the large amount of ultramarine injected, so little could be recognized after it had left the abdomen. It regularly disappeared until finally, except for a few stained bronchial glands and $a$ blue deposit coloring the diaphragm, none could be found.

This may be due to the relatively large dilution of the solid matter by the body fluids or to its rapid elimination or to chemical alteration of the substance after prolonged contact with the body fluids.

I have therefore decided to repeat the experiments, using a stable substance which can not possibly form the basis of a natural anthracosis and which can be easily separated unchanged and recognized.

Talc seems ideal for this purpose. Unchanged by high temperatures and extremely stable chemically, its separation from the organs becomes a matter of merely following out a certain technic and its recognition is simple and absolute.

By injecting weighed amounts of talc into the peritoneal cavity or alimentary canal and later separating the substance from the various organs and excreta by combustion, treatment with acids, etc., the amount of the deposit occurring in the lungs and other organs, as well as the amount eliminated, can be definitely settled. The result of these experiments will form the basis of a later paper.

240 Stockton Street.

\section{A CASE OF OVARIAN PREGNANCY}

\section{N. S. MAC DONALD, M.D. HANCOCK, MICH.}

The following condition is reported mainly on account of its comparative rarity and to substantiate further the claim made by many authorities that in certain cases of ectopic pregnancy rupturing into the free abdominal cavity the patient may recover if left alone.

History.-On March 27, 1908, was called to see a primipara, who presented the usual characteristic symptoms of ruptured extrauterine pregnancy. She had missed one period. A hot saline enema was given and the patient was removed to St. Joseph's hospital.

operation.-Four hours later, her condition having improved somewhat, the abdomen was opened under ether anesthesia and found filled with clotted blood, but nowhere were there signs of active hemorrhage. On passing the hand into the cul-de-sac and along both tubes, the only unusual thing noted was that the right ovary seemed to be somewhat enlarged, of soft consistency and collapsible. On bringing the ovary into the wound it was found to be cup-shaped, of about three times normal size and hollowed out from above to the degree that only $x$ thin layer of ovarian tissue remained to form the wall. The cavity within the ovary contained a small clot, after the removal of which slight oozing was noticeable. The. ovary was removed on principle, the clots washed out and the abdominal cavity filled with hot saline solution previous to closure.

Result.-The patient made a good recovery and left the hos. pital within three weeks.

\section{A SUGGESTION FOR MORE READILY DETER- MINING INJURIES OF THE URETER DURING OPERATION \\ HENRY DAWSON FURNISS, M.D. NEW YORK}

Having within the past year been called on to use the cystoscope on three patients with injured ureters, it occurred to me that the following method might be of assistance in detecting these injuries at the time of operation. The surest method is to pass bougies or eatheters into the ureters, but many times this is impracticable, both on account of conditions presented by the patient, and lack of experience on the part of the operator. Often the ureter may be cut, the cut portion inspected, and then there be doubt as to its identity. It is very easy to give a patient methylene blue by mouth before operation, or to inject into the gluteal muscles at the time of operation 20 minims of a 0.4 per cent. aqueous solution of indigo-carmine. These drugs will so color the urine that, should the ureter be cut and there be any leakage from it, the colored solution would be promptly recognized. If the ureter were cut on the distal side of a clamp or ligature, there might be enough discoloration so that it could then be recognized. It is fully realized that many injuries may occur to the ureter in which this procedure would be of no account, but it is so simple, and would at times be of such help, that I think it should be used often.

393 West End Avenue.

Macular and Perimacular Hemianopic Scotoma Following Knife Wound of the Occipital Lobe.-Henschen reports, in Hygiea Festband, a case of limited traumatic lesion of the occipital lobe causing a macular scotoma. He holds that the case proves that the macula has a distinct and limited center in the occipital lobe, which center he would designate as a cortical retina. 